\title{
Postsynaptic Modulation of Rectifying Electrical Synaptic Inputs to the LG Escape Command Neuron in Crayfish
}

\author{
Donald H. Edwards, ${ }^{1}$ William J. Heitler, ${ }^{1, a}$ Esther M. Leise, ${ }^{1, b}$ and Russell A. Fricke ${ }^{2, c}$ \\ 'Department of Biology, Georgia State University, Atlanta, Georgia 30303 and ${ }^{2}$ Department of Anatomy and Cell Biology, \\ Emory University School of Medicine, Atlanta, Georgia 30322
}

The lateral giant (LG) tail-flip escape system of crayfish is organized to provide a massive convergence of mechanosensory inputs onto the LG command neuron through electrical synapses from both mechanosensory afferents and interneurons. We used electrophysiological techniques to show that the connections between three major mechanosensory interneurons and LG rectify, and that their inputs to LG can be reduced by postsynaptic depolarization and increased by postsynaptic hyperpolarization. The mechanosensory afferents and interneurons are excited by sensory nerve shock, and the components of the resulting LG PSP can be similarly modulated by the same postsynaptic potential changes. Because these inputs are all made through electrical synapses, we conclude that they are rectifying connections, as well. To test the physical plausibility of this conclusion, we developed an electrical model of the rectifying connection between a mechanosensory interneuron and LG, and found that it can reproduce all the qualitative features of the orthodromic and antidromic experimental responses.

The ability of postsynaptic membrane potential to modulate inputs through rectifying electrical synapses is used in the escape system to enhance LG's relative sensitivity to novel, phasic stimuli. Postsynaptic depolarization of LG produced by earlier inputs "reverse-biases" the rectifying input synapses and reduces their strength relative to times when LG is at rest.

Electrical synapses provide for rapid and reliable transmission between neurons, but at the expense of unidirectionality and plasticity. Rectifying electrical synapses combine some of the advantages of both standard electrical and chemical synapses. They transmit rapidly by allowing charge to flow directly from the pre- to the postsynaptic cell, but only when the presynaptic neuron is more positive than the postsynaptic cell. This rectification property, which was first described in crayfish at the

\footnotetext{
Received Nov. 20, 1990; revised Feb. 14, 1991; accepted Feb. 15, 1991

We would like to thank Drs. F. B. Krasne and E. Vu for many thoughtful discussions of this work, which was supported by NIH Research Grant NS26457.

Correspondence should be addressed to Dr. Donald Edwards, Department of Biology, Georgia State University, Atlanta, GA 30302-4010.

a Present address: Department of Biology and Preclinical Medicine, Gatty Marine Laboratory, University of St. Andrews, St. Andrews, KY16 8LB, Scotland.

b Present address: Kewalo Marine Laboratory, 41 Ahui Street, IIonolulu, HI 96813.

' Present address: St. Clare's Hospital, 600 McClellan Street, Schenectady, NY 12308.

Copyright (C) 1991 Society for Neuroscience $0270-6474 / 91 / 112117-13 \$ 03.00 / 0$
}

giant motor synapse (Furshpan and Potter, 1959a), gives these electrical synapses the unidirectional transmission characteristic common to chemical synapses. In crayfish, rectification prevents the giant motor neuron (MoG) from antidromically exciting its driver neurons, the lateral giant (LG) and medial giant (MG) interneurons, that separately excite it through adjacent rectifying electrical synapses.

A less recognized quality of rectifying electrical synapses is that their transmission can be modulated by changing the membrane potential of the postsynaptic cell (Furshpan and Potter, 1959b; Friesen, 1985; Giaume et al., 1987). Rectifying electrical postsynaptic potentials (PSPs) and postsynaptic currents (PSCs) in $\mathrm{MoG}$ are increased in amplitude when the cell is hyperpolarized and decreased when it is depolarized (Furshpan and Potter, 1959b; Edwards, 1990a).

Synaptic transmission that is high-speed, reliable, unidirectional, and modulatable is of obvious use in the crayfish escape circuit, which initiates the animal's tail flip away from a predator within $15 \mathrm{msec}$ of contact (Wine and Krasne, 1982). The rectifying electrical giant motor synapse between LG and MoG is part of that circuit, which also contains many other electrical synapses (Wine and Krasne, 1982). On the afferent side of the circuit, $L G$ receives input through electrical synapses from primary afferents and interneurons that are excited by mechanosensory stimuli (Zucker, 1972). If the electrical synapses between mechanosensory afferents or mechanosensory interneurons (MSIs) and LG were ohmic (nonrectifying), we would expect that LG might antidromically excite the afferent network in segments away from the source of the LG impulse. This fails to happen despite a favorable size relationship between $L G$ and all the mechanosensory afferents and MSIs, which led us to conclude that the electrical synapses between LG and its inputs are likely to rectify.

We present evidence here that the electrical synapses onto LG from mechanosensory afferents and MSIs do rectify. We also show that tonic depolarization of $L G$ reduces transmission through its rectifying input synapses by reducing both the transsynaptic potential difference and the period of high transsynaptic conductance produced by a presynaptic impulse. This mechanism also works at the rectifying giant motor synapse when a depolarizing IPSP (d-IPSP) in MoG reduces input from LG (Hagiwara, 1958; Furshpan and Potter, 1959b; Edwards, 1990a). Tonic depolarization of LG occurs as a late response to phasic mechanoscnsory input (Krasne, 1969), and it has the effect of reducing the cell's response to late inputs. This mechanism of depolarizing inhibition works together with another, more effective chemical synaptic inhibition to prevent LG from re- 
$\mathbf{A}$

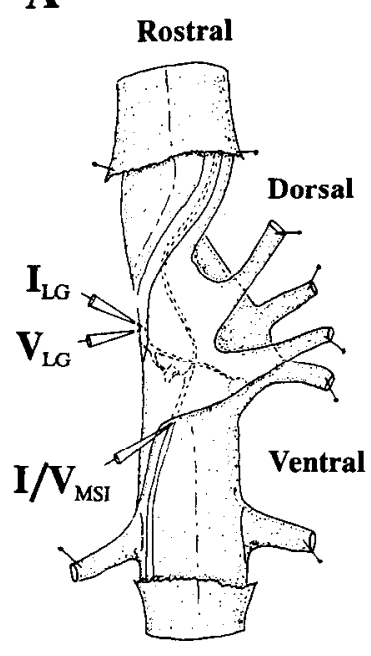

Caudal
B

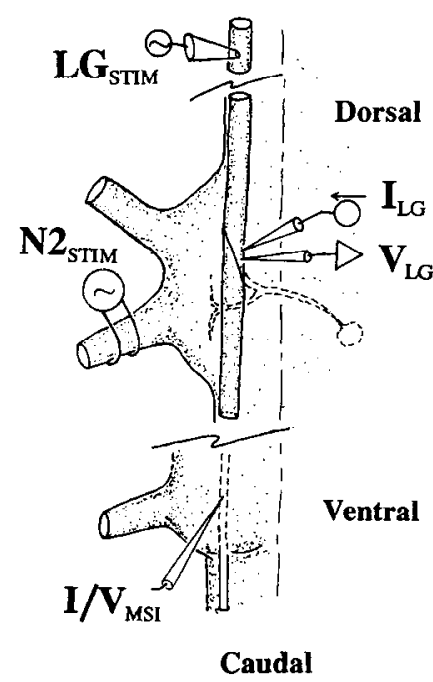

Figure 1. Diagrams of physiological preparations. A, Arrangement for recording from an MSI and LG in the same hemi-segment. Caudal to the ganglion, the isolated nerve cord was pinned out ventral side up, whereas rostral to the ganglion the nerve cord was pinned out dorsal side up. The ganglion was folded to expose the dorsal aspect of the right side and the right LG, and the ventral aspect of the right side and the MSI axons at the caudal margin of the ganglion. Solid lines indicate visible features, for example, LG and MSI axons, and broken lines indicate the interior structures, for example, putative MSI/LG contact sites. Two electrodes were placed in the initial axon segment of LG for current injection and recording $\left(I_{L G}\right.$ and $V_{L G}$ ), and a third electrode was in the MSI axon at the caudal margin of the ganglion $\left(I / V_{M S I}\right) . B$, Arrangement for recording an antidromic synaptic potential in an MSI in response to an LG impulse, and for recording $L G$ responses to current injection and nerve shock. Antidromic LG impulses were excited by 0.25 -msec electrical pulses delivered to the $L G$ axon by an en passant suction pipette $\left(L G_{\text {STIM }}\right)$. MSI antidromic synaptic potentials were recorded in response to LG stimulation by a ganglionic micropipette $(I /$ $\left.V_{M S I}\right)$, when the MSI was responding to injected current through the recording electrode. Current injection and voltage recording micropipettes $\left(I_{L G}\right.$ and $\left.V_{L G}\right)$ were inserted into LG's initial segment exposed on the dorsal ganglionic surface, and sensory afferents in the ipsilateral ganglionic nerve 2 were excited by electrical stimulation of the nerve $\left(N 2_{\text {STIM }}\right)$.

sponding to anything but very phasic mechanosensory stimuli (E. T. Vu and F. B. Krasne, unpublished observations).

\section{Materials and Methods}

Adult crayfish (8-12-cm Procambarus clarkii) were obtained from a commercial supplier (Waubun Laboratories, Schriever, LA) and kept in laboratory aquaria until used. After the experimental animal was anesthetized in ice water, the abdominal nerve cord was removed and pinned out in a Petri dish lined with Sylgard (Dow-Corning). The nerve cord was covered with aerated, chilled saline (Van Harreveld, 1936) and desheathed in those segments where intra- or extracellular recordings were to be made. The preparation was allowed to equilibrate gradually to room temperature, which was kept near $18^{\circ} \mathrm{C}$.

Electrophysiology. Intracellular potentials were recorded by standard means. Current was injected into LG through a low-resistance $(<10$ $\mathrm{M} \Omega$ ) microelectrode attached to a high-current headstage of a preamplifier (Axon Instruments). Membrane potentials from the mechanosensory interneurons and LG were recorded through higher-resistance $(\sim 20 \mathrm{M} \Omega)$ microelectrodes and measured against a bath reference potential established by a virtual ground circuit. Amplified signals were digitized, displayed, and stored on magnetic tape (Neurodata Corp.) and later analyzed using pCLAMP software (Axon Instruments). Mechanosensory afferents and mechanosensory interneurons were excited by

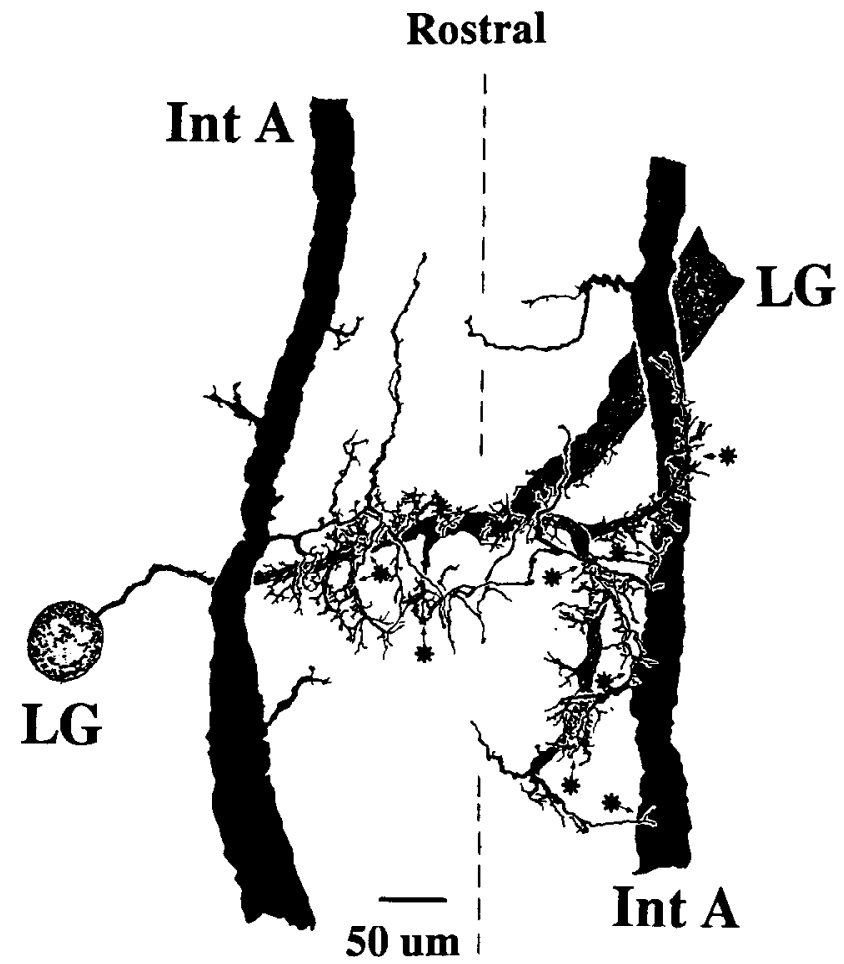

Caudal

Figure 2. Int A and its putative contact sites with LG. Camera lucida drawings of cobalt-filled left and right Int $A$ axons and right LG in G5, and their probable sites of contact (asterisks). The broken line indicates ganglionic midline.

electrical stimuli applied to sensory nerves by suction pipettes driven by a stimulus-isolated pulse generator (Grass Instruments). Mechanosensory interneurons were identified by their responses to sensory nerve stimulation and by their shape as revealed by Lucifer yellow dye injected into the cell by iontophoresis from the recording microelectrode (Kennedy, 1971; Stewart, 1978; Sigvardt et al., 1982).

Three different preparations were used. To record the properties of synapses between a mechanosensory interneuron and LG, electrodes were placed in both neurons in the samc ganglion (G3 or G4), as in Figure $1 A$. The nerve cord was twisted at the ganglion, so that all four ganglionic nerves were pinned unilaterally and the dorsal aspect of the cord was exposed on the other side. Two micropipettes were placed under visual guidance in the exposed initial axon segment of the right LG. The group of large, ventrolateral mechanosensory interneuron axons was exposed at the caudal margin of the ganglion, and one axon was found that responded to stimulation of the ipsilateral fourth nerve in G6 (G6N4) and evoked PSPs in LG. Two mechanosensory interneurons, Int $A$ and Int $C$, were identified from their receptive fields, their different responses to sensory nerve shock, and their large and distinctively branching axon terminals in rostral segments (Kennedy, 1971; Zucker, 1972; Sigvardt et al., 1982).

To record synaptic responses to shock of a ganglionic nerve, two micropipettes were placed in the initial scgment of the ipsilateral LG, one to inject current and the other to record voltage responses (Fig. 1B).

Antidromic synaptic potentials were recorded in a mechanosensory interneuron that was penetrated within one ganglion $(\mathrm{G} 3, \mathrm{G} 4$, or $\mathrm{G} 5$; Fig. $1 B$ ), while the LG spike was monitored intracellularly from the axon near a rostral ganglion.

Neuronal morphology. Possible sites of synaptic contact between LGs and mechanosensory interneurons were identified by marking both neurons with cobalt sulfide intensified by Timm's procedure (Obermayer and Strausfeld, 1980). Cells were viewed in whole-mount preparations and drawn with a camera lucida.

Computer simulations. The compartmental model of the MSI/LG synapse and the simulations using that model were both carried out 
$\mathbf{A}$

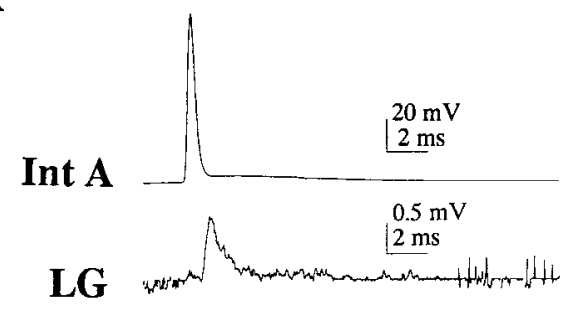

B

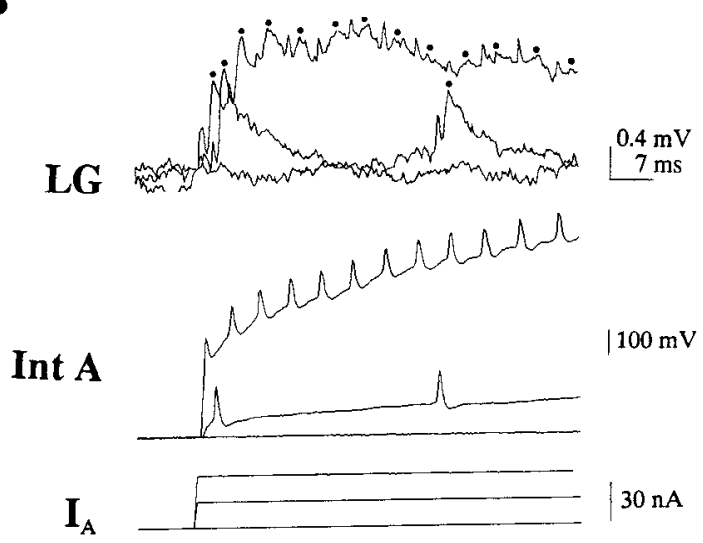

$\mathbf{C}$
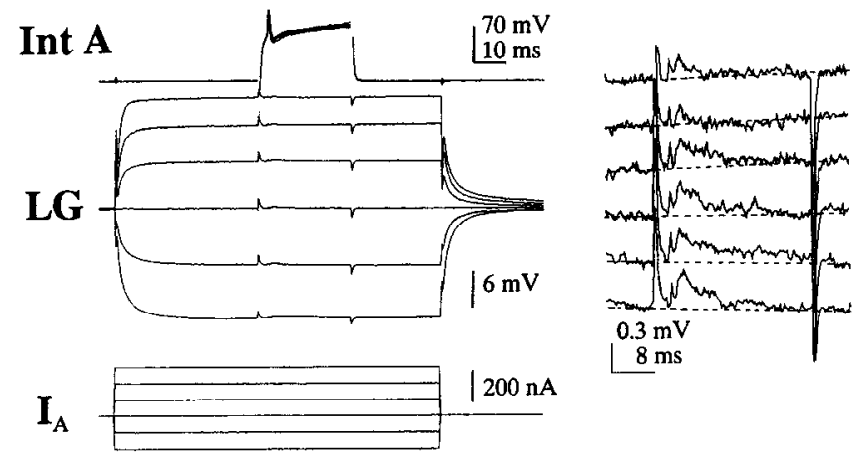

D
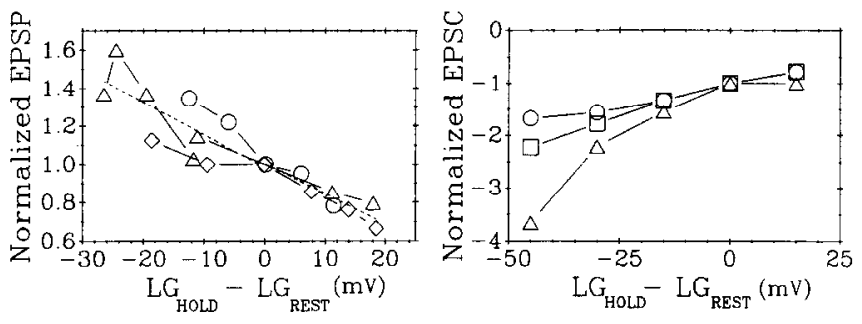

Figure 3. Orthodromic transmission between ipsilateral Int A and LG. $A$, Int $A$ impulse in G3 and EPSP in G2 $L G$ evoked by shock of G6N4. $B$, Responses of $L G$ and Int $A$ in G4 to depolarizing and hyperpolarizing current injected into Int $\mathrm{A}$; Int $A$ responses were recorded with the same electrode through a balanced bridge. The small voltage pulses in the LG record are coupling artifacts from the Int A spikes; each immediately precedes the EPSP (circles) evoked by that spike. C. Current into LG (bottom left) shifts LG's membrane potential (middle left) and modulates EPSPs (middle left and amplitude traces on right) evoked by Int A impulses (top left) triggered by depolarizing current pulses. Broken lines indicate approximate baselines. $D$, Normalized EPSP amplitudes evoked by Int A in three LG neurons (left; identified by various $s y m b o l s$ ) and normalized EPSC amplitudes from three more preparations (right) plotted against the difference between the LG holding potential and rest potential ( -85 $\mathrm{mV}$ ). Ordinate values are normalized to EPSP and EPSC amplitudes when LG is at rest. Two regression lines (broken lines) are shown for combined EPSP data recorded during LG hyperpolarizations and LG depolarization; the lines are very nearly colinear.

with the simulation program NEURON, which is freely available from D. H. Edwards upon request (Edwards and Mulloney, 1984, 1987; Edwards, 1990b). The program was run on a Compaq 386/20 microcomputer.

\section{Results}

Rectification between Int $A$ and $L G$. The Int $\mathrm{A}$ [also known as A6 (Wine and Krasne, 1982) and 6B1 (Wine, 1984)] interneurons are a bilaterally homologous pair of cells that are the largest MSIs. Each receives input only from ipsilateral mechanosensory afferents in G6 and contacts LG in all rostral abdominal ganglia (Kennedy, 1971; Zucker, 1972). In G5, these contacts appear to be made at intervals along the rostral and caudal branches of the major LG dendrite that is ipsilateral to both the LG axon and Int A (Fig. 2). A few contacts also appear to be made by axonal branches of Int A onto the contralateral dendrite of LG. The contralateral Int $A$ appears to make only a few contacts on that contralateral LG dendrite.

Phasic mechanosensory stimulation of the tail fan or shock of the ipsilateral G6N4 both excite from one to three Int A impulses at short latency (Kennedy, 1971; Fig. 3A). Each impulse originates in G6, has an overshooting peak and a prolonged depolarizing afterpotential, and produces a fast-rising EPSP in the LG of each ganglion through which it passes.
We were able to stimulate Int A with injected current through a ganglionic electrode in four preparations where we could also record the postsynaptic response of $\mathrm{LG}$ (Fig. $1 A$; see Materials and Methods). Int A spikes excited by low levels of depolarizing current evoked LG EPSPs (Fig. 3B, circles) that lasted considerably longer than EPSPs evoked by conducted impulses (Fig. $3 A$ ). The increased EPSP duration is presumably caused by the presynaptic depolarization produced by the injected current. Higher levels of depolarizing current triggered high-frequency trains of Int A spikes. The resulting LG EPSPs became smaller as they summed to produce a sustained depolarization of LG. Hyperpolarizing currents injected into Int A produced small changes in LG's membrane potential.

Shifts in the LG's membrane potential could modulate PSPs and PSCs produced by impulses in Int $\mathrm{A}$. Int $\mathrm{A}$ impulses were triggered by pulses of depolarizing current, while $L G$ responded to steps of hyperpolarizing and depolarizing current applied at the initial axon segment by a two-electrode current clamp (Fig. $3 C)$. In three preparations, EPSP amplitude varied linearly with the shift in LG's membrane potential (Fig. 3D). The sensitivity of EPSPs to membrane potential shifts is given by the slopes of regression lines of the normalized EPSP amplitudes plotted against the shift in membrane potential. They show that EPSP amplitudes increased by $1.7 \% / \mathrm{mV}$ of hyperpolarizing shift and 


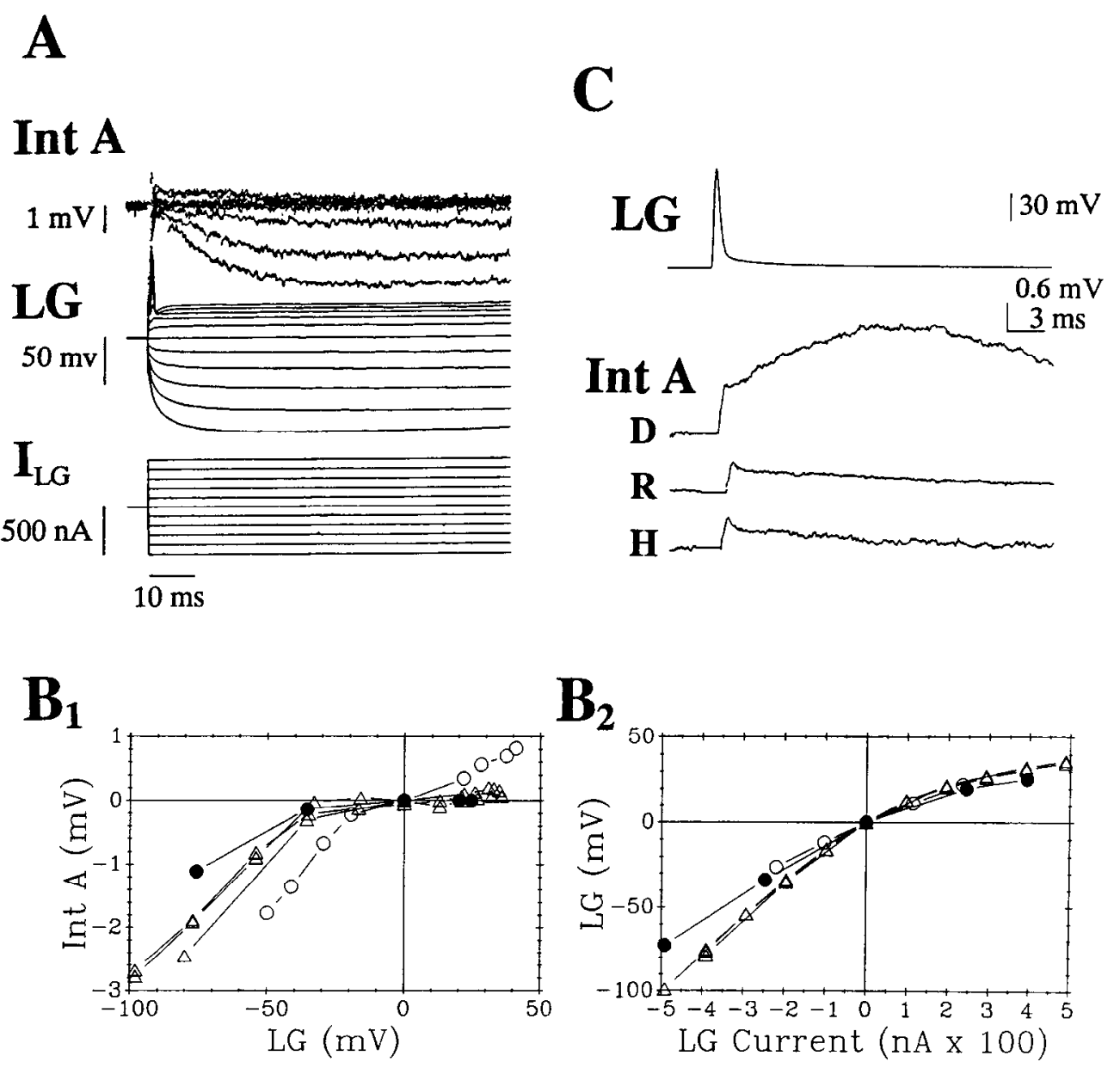

Figure 4. Antidromic potentials in Int A produced by impulses and DC potential shifts in LG. $A$, Spread of hyperpolarization, but not depolarization, from LG to Int A in G4. $B_{i}$, Plot of membrane potential shift in Int A versus potential shift in LG produced by current injection. Data from three preparations are identified by different symbols. $B_{2}$, Plots of LG potential shift versus injected current for the same preparations. C, LG impulses (top trace, recorded from $L G$ axon near $G 2$ ) evoked antidromic synaptic potentials in right G3 Int A (lower three traces) that increased from the value at rest $(R)$ when Int $\mathrm{A}$ was depolarized $(D)$ and decreased when LG was hyperpolarized $(H)$ by current injection through the recording electrode. Averaged responses to five stimuli are shown. The levels of depolarization and hyperpolarization were uncertain because of the voltage change produced by the electrode resistance (see Results).

50


ponent of the antidromic synaptic potential relative to its amplitude at rest, and hyperpolarization decreased it. When data from all preparations are combined, antidromic synaptic potentials recorded in Int $A$ at rest had an average initial peak amplitude of $0.30 \pm 0.12 \mathrm{mV}$ (mean $\pm \mathrm{SD}$ ). The peak amplitude increased by $164 \pm 79 \%$ when the cell was depolarized and decreased by $53 \% \pm 27 \%$ when Int $A$ was hyperpolarized.

More striking was the effect of depolarization on the wave form of the antidromic synaptic potential. Instead of a slow decline from the initial peak, which occurred at rest and during hyperpolarization, depolarization of Int A caused the antidromic synaptic potential to display a large, slow wave of depolarization that lasted for the duration of the depolarizing afterpotential following the LG spike. Presumably, the presynaptic depolarization produced by the injected current increased the coupling between the cells and allowed the depolarizing afterpotential to pass antidromically. Also contributing to the depolarizing afterpotential in LG, and thereby to the antidromic synaptic potential in Int $\mathrm{A}$, is a recurrent depolarizing IPSP that is triggered in each ganglionic I.G by an I G impulse (Roherts, 1968). This depolarizing IPSP is reduced in the record of the LG impulse in Figure $4 C$, which was obtained from an axonal recording near $\mathrm{G} 2$.

Rectification between Int $C$ and $L G$. Int $C$ [also known as A64 (Wine and Krasne, 1982)] is a higher-order MSI that is excited by mechanosensory afferents, by Int $\mathrm{A}$, and by other MSIs in each abdominal segment (Kennedy, 1971; Calabrese and Kennedy, 1974; Sigvardt et al., 1982). It has an extensive arborization in G4, G5, and G6; we have not examined the more rostral segments. In G5, part of that arborization appears to contact the major ipsilateral and contralateral LG dendrites at several locations along their length (Fig. 5).

Int $\mathrm{C}$ fired spontaneously at a low frequency in an isolated nerve cord. Each impulse rose from a rest potential $(-83.3 \pm$ $3.7 \mathrm{mV} ; n=7)$ similar to Int A's and repolarized after a 10$\mathrm{mV}$ depolarizing afterpotential that lasted for more than 20 msec (Fig. 6A). The EPSPs evoked in LG were similar to those evoked by Int $A$ : in each case, a rapid depolarization was followed by a slow repolarization.

Int $\mathrm{C}$ responded to phasic mechanosensory stimulation of the abdomen or to sensory nerve shock with a long-lasting, highfrequency discharge (Fig. 6B; Kennedy, 1971; Sigvardt et al., 1982). The depolarizing afterpotentials summated during that discharge so the impulses rose from a sustained depolarization that exceeded $10 \mathrm{mV}$ and lasted for more than $30 \mathrm{msec}$. As with repetitive excitation of Int $\mathrm{A}$, high-frequency discharge of Int $\mathrm{C}$ evoked by root shock produced EPSPs in LG that summated as they tonically depolarized the cell (Fig. 6C).

We studied the effects of both pre- and postsynaptic potential changes on the coupling between Int $C$ and $L G$ in seven preparations. As with Int A (Fig. 3C), hyperpolarization of $L G$ increased the amplitude of F.PSPs evoked by Int C, and depolarization decreased them (Fig. $6 C ; E$, left). Depolarization of $\mathrm{LG}$ decreased the PSP amplitude by about $-1 \% / \mathrm{mV}$ change in $\mathrm{LG}$ potential, and hyperpolarization increased the amplitude by $+0.75 \% / \mathrm{mV}$. The sensitivity of LG's response to membrane potential shifts increased at later times as the PSPs summated. At $21 \mathrm{msec}$ after the onset of the first PSP (Fig. 6C, *), the summated PSP sensitivity to hyperpolarization had increased to $+2.1 \% / \mathrm{mV}$, and the sensitivity to depolarization was $-2.5 \%$ / $\mathrm{mV}$. The input resistance of LG changed very little over all but the most depolarized end of the voltage range, and it and the

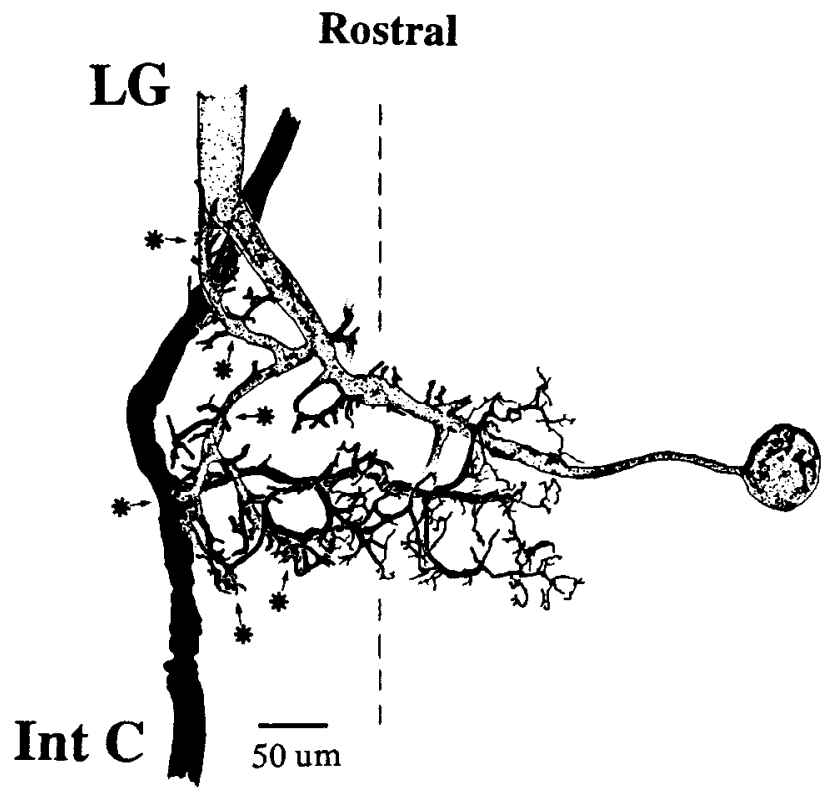

\section{Caudal}

Figure 5. Int $\mathrm{C}$ and possible contact sites with ipsilateral LG in G5. Camera lucida drawings of left LG and Int C in G5, and the part of Int C's arbor where contacts between the cells are possible (asterisks). The broken line indicates ganglionic midline.

cell's charging curves were constant over the hyperpolarizing end of the range. We infer that changes in postsynaptic voltagesensitive conductances did not occur over this range and cannot account for the effect of hyperpolarization on EPSP amplitude.

The early rapid and late slow components of LG's response to Int $\mathrm{C}$ impulses are emphasized when a two-electrode voltage clamp is used to record EPSCs in LG rather than EPSPs. Each Int $C$ spike produced a large, phasic inward current (Fig. 6D, P) in LG followed by a smaller, slower inward current (S). Hyperpolarization of $L G$ increased the amplitudes of both response components, and depolarization reduced them (Fig. $6 D ; E$, right). Finally, impulses in LG evoked antidromic synaptic potentials in Int $C$ that were enhanced when Int $C$ was depolarized and reduced when Int $\mathrm{C}$ was hyperpolarized, in identical fashion to those seen in Int A (Fig. 4C).

Rectification between an unidentified MSI and LG. The number of MSIs that contact LG is uncertain; the one other that we tested also displayed rectification in its contact with LG. We recorded the response of this unidentified MSI in ganglion G4 to shock of sensory nerves in G6 and found that the cell's impulses evoked PSPs in LG that summated with those from other sources. Two additional electrodes placed in the initial segment of the ipsilateral LG axon in G4 allowed us to voltage-clamp LG to different holding potentials and to record PSCs in response to MSI spikes like those from Int C (Fig. 6D). The PSCs increased in amplitude when LG was hyperpolarized, and decreased when it was depolarized (not shown). We also recorded both cells' responses to current injected into LG, and their responses to current injected into the MSI. Transmission through this synapse rectified in the same manner as the Int A/LG synapse. We found that a depolarization of the MSI spread more effectively into LG than did a larger hyperpolarization produced by the same amount of current. Conversely, we found that hy- 


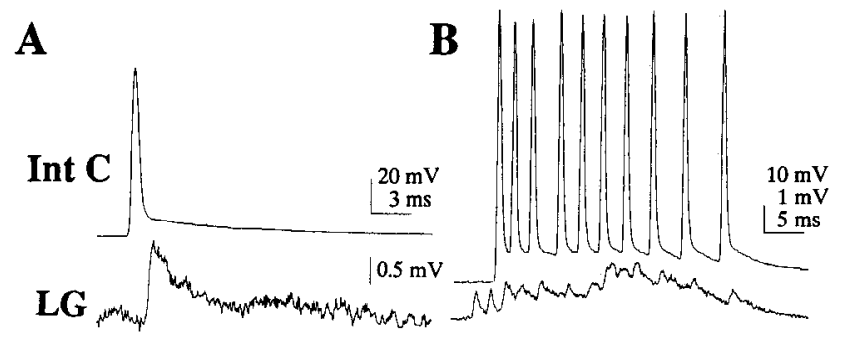

$\mathbf{C}$
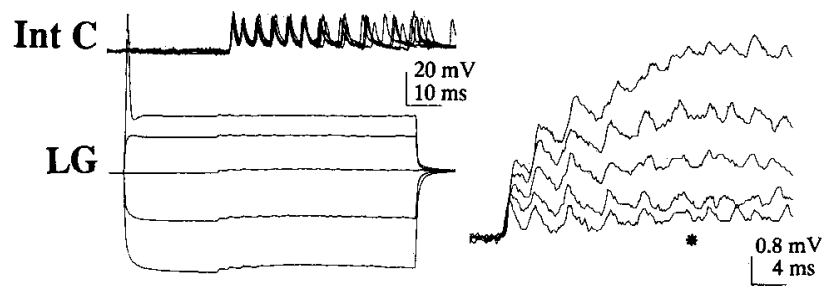

$\mathbf{I}_{\text {LG }}$

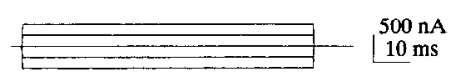

$\mathbf{D}$

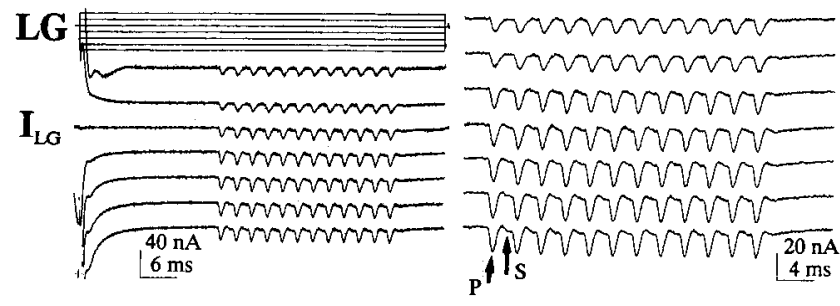

$\mathbf{E}$
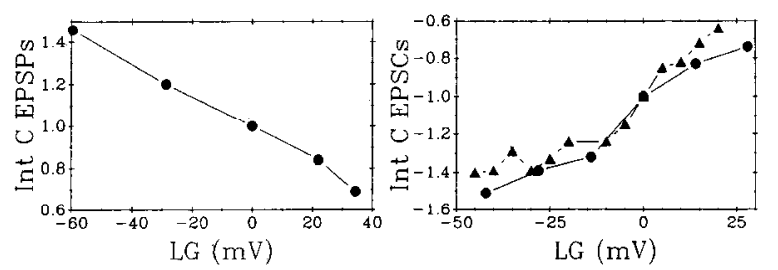

Figure 6. Rectification between Int C and LG. A, A single LG impulse in G5 (top trace) and resulting LG PSP in G3 (bottom trace). B, Response of Int C and LG to strong shock of a sensory nerve. First two PSPs in the bottom trace were produced by Int A spikes; later PSPs correlated one to one with Int $C$ spikes. $C$, LG hyperpolarization increases and depolarization decreases LG's responses to Int C. Top, Trains of attenuated Int C spikes (from a poor penetration) in left G3 in response to single shocks of right G6N4. Middle, LG PSPs in left G6 LG during response to current injection (bottom). Right, PSPs aligned and amplified. The asterisk indicates the time at which EPSP sensitivity to LG membrane potential was calculated (see Results). D, Two-electrode voltage-clamp records of EPSCs in LG (bottom left) produced by repetitive stimulation of Int C as LG is held at potentials from $-40 \mathrm{mV}$ to $+20 \mathrm{mV}$ from rest (top left). EPSCs are amplified and aligned at the right (different preparation from $C$ ). The arrows indicate phasic $(P)$ and slow $(S)$ inward currents of an EPSC. $E$, Plot of the normalized EPSP $(l e f t$; from $C)$ and EPSC $($ right; from $D$ and one other preparation; indicated by different $s y m b o l s)$ amplitudes versus shift in LG membrane potential from rest.

perpolarizing potentials in LG spread readily into the MSI, whereas depolarizing potentials did not. The coupling between the two cells increased when LG was hyperpolarized by more than $5 \mathrm{mV}$. Because the rest potential of the MSI was $-70 \mathrm{mV}$,

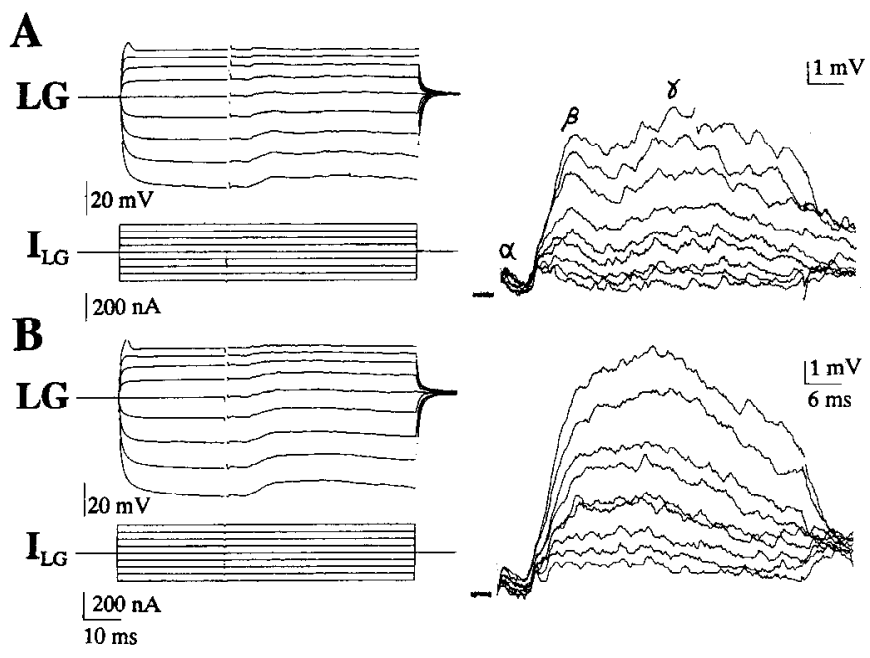

Figure 7. Modulation of compound synaptic potentials in LG by membrane potential changes. $A$, EPSPs in right $\mathrm{G} 6 \mathrm{LG}$ in response to shock of ipsilateral G6N4 recorded during hyperpolarization and depolarization of LG (top left) by injected current (bottom left). EPSPs are aligned and amplified at right; the largest EPSPs occurred when LG was hyperpolarized, whereas the smallest occurred when LG was depolarized. $\alpha$-, $\beta$-, and $\gamma$-components are indicated. $B$, The same experiment after $10 \mu \mathrm{M}$ picrotoxin eliminated the postexcitatory inhibition that separates the $\beta$ - and $\gamma$-components of the root-evoked EPSP. Records are presented as in $A$. which is $15 \mathrm{mV}$ more positive than LG, the rectification threshold for the synapse was approximately $20 \mathrm{mV}$.

Modulation of sensory nerve-evoked PSPS in LG by postsynaptic membrane potential. Shock of a ganglionic sensory nerve evokes a compound PSP in LG produced by primary mechanosensory afferents and MSIs (Fig. 7; Krasne, 1969). The early, $\alpha$-component is produced by the mechanosensory afferents and occurs 2-3 msec after the nerve stimulus. The later, $\beta$-component is produced by a disynaptic pathway through the MSIs, including Int A and Int C (Zucker, 1972), whereas the still later $\gamma$-component is produced by polysynaptic pathways. As before, hyperpolarization of LG increases the amplitudes of all components of the PSP, whereas depolarization reduces them (Fig. $7 A)$.

Sensory nerve shock also leads to a postexcitatory inhibition of LG and the MSIs that excite it; together these produce the dip in the EPSP amplitude that separates the $\beta$-component from the later $\gamma$-component (Krasne et al., 1990; Vu and Krasne, unpublished observations). Postexcitatory inhibition is sensitive to picrotoxin (F. B. Krasne, personal communication); we found that addition of $10 \mu \mathrm{M}$ picrotoxin to the bath abolished the dip in the LG PSP, but did not change the effect of LG hyperpolarization and depolarization on the larger root-evoked EPSP (Fig. 7B).

The $\alpha$-, $\beta$-, and $\gamma$-components are similarly affected by shifts in the LG membrane potential. Plots of the normalized amplitudes of the different PSP components against LG membrane potential from seven preparations are shown in Figure 8. As before, the sensitivity of the PSP components to LG membrane potential changes is given by the slope of regression lines calculated for each data set. The sensitivity of the normalized 

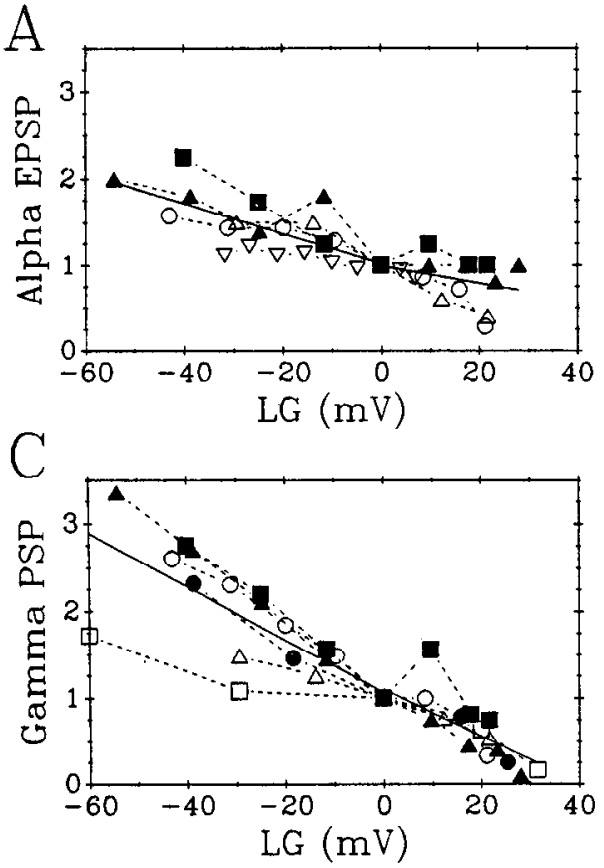

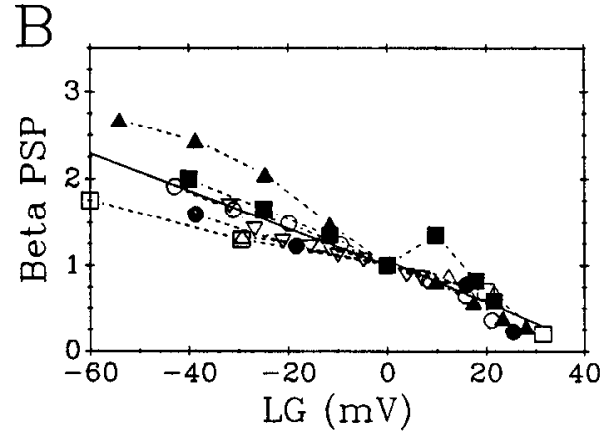

Figure 8. Plots of the normalized amplitudes of nerve-evoked EPSP components in $L G$ versus $L G$ membrane potential shift from rest. $A, \alpha$-Components. $B, \beta$-Components. $C, \gamma$-Components. Data are from seven preparations, each represented by a different symbol connected by broken lines. The two solid lines in each plot are linear regressions of EPSP components from all animals recorded during hyperpolarizing and depolarizing shifts $C$, respectively.
$\alpha$-PSPs to hyperpolarization of $\mathrm{LG}$ was $+1.8 \% / \mathrm{mV}$, and to depolarization it was $-1.0 \% / \mathrm{mV}$. The sensitivity of the $\beta$-PSP to hyperpolarization was $+2.2 \% / \mathrm{mV}$, and to depolarization it was $-2.4 \% / \mathrm{mV}$. The sensitivity of the peak $\gamma$-response was greatest: it was $+3.0 \% / \mathrm{mV}$ to LG hyperpolarization and $-2.75 \%$ / $\mathrm{mV}$ to $\mathrm{LG}$ depolarization.

A simple electrical model of rectifying inputs to $L G$. The experimental results presented above show that the electrical synapses between Int $A$ and LG and between the unknown MSI and LG rectify. They also showed that LG EPSPs and antidromic synaptic potentials in Ints $A$ and $C$ can be modulated by shifts in their membrane potentials. A recent study of the giant motor synapse showed that similar modulation of MoG's EPSP occurs when that cell's membrane potential is changed, and that a significant part of the modulatory effect results from changes in the transsynaptic conductance of the rectifying electrical synapse (Edwards, 1990a,b). We wished to determine whether a simple electrical model of a rectifying synaptic connection between an MSI and LG could account for the potentialdependent modulation of electrical synaptic transmission seen here.

The model contains seven electrical compartments, six of which represent the postsynaptic structure of $L G$, and the other, the presynaptic MSI (Fig. 9A). The six LG compartments represented a length of gradually widening dendrite (Fig. 9A, compartments 1-3) attached to a large, low-resistance axon (compartments 4-6). The presynaptic compartment generated action potentials that evoked PSPs in the LG model, and produced antidromic synaptic potentials in response to antidromic impulses in the axon of the LG model. The action potentials were produced by sodium, potassium, and leakage currents governed by the Hodgkin-Huxley equations (Hodgkin and Huxley, 1952), with the addition of a delayed conductance in series with a reversal potential of $+20 \mathrm{mV}$ from rest to produce the depolarizing afterpotential. The values of the conductances and reversal potentials are given in Table 1 . The resting conductances and reversal potentials enabled the LG compartments to have a rest potential $4 \mathrm{mV}$ more negative than the MSI compartment, much as LG is $4 \mathrm{mV}$ more negative at rest than Int $A$. The rectifying synaptic conductance $\left(g_{s}\right)$ between the MSI compartment and the distal dendritic compartment (compartment 1) of the LG model was described by Equation 1:

$g_{s}=g_{\min }+\left\{\left(g_{\max }-g_{\min }\right) /\left(1+\exp \left[A\left(V_{\mathrm{MSI}}-V_{\mathrm{LG}}-V_{\mathrm{o}}\right)\right]\right)\right\}$,

where $g_{\min }$, the minimal transsynaptic conductance, equals 0.2 $\mu \mathrm{S} ; g_{\max }$, the maximal conductance, equals $5 \mu \mathrm{S}$; the exponential coefficient, $A$, equals $-1 / \mathrm{mV}$; and the rectification threshold, $V_{0}$, equals $25 \mathrm{mV}$. A very similar form of the same equation was used previously to describe the voltage-dependent conductance of the giant motor synapse (Giaume et al., 1987; Edwards, 1990b). The kinetics of the rectifying electrical conductance at the giant motor synapse have been measured and were found to have opening and closing time constants of $7 \mathrm{msec}$ at $9.4^{\circ} \mathrm{C}$ and a $Q_{10}$ of 11 (Jaslove and Brink, 1986). We assumed that the rectifying electrical synapse between the MSI and LG has similar kinetics, and we calculated that the opening and closing time constants should equal $0.8 \mathrm{msec}$ at $18^{\circ} \mathrm{C}$, the approximate temperature of the experimental preparations. In the model, changes in the rectifying synaptic conductance that occur in response to a change in transsynaptic potential follow first-order kinetics governed by these time constants (Edwards, 1990b).

Postsynaptic polarization of model compartments modulates PSPS. Polarization of postsynaptic LG compartments alters the amplitude and wave form of PSPs evoked by impulses in the presynaptic MSI compartment. An action potential that ends with a depolarizing afterpotential was triggered in the MSI compartment when the LG model was responding to current injected into compartment 3 (Fig. $9 B$ ), which corresponds to the most proximal segment of the LG dendrite (Fig. 9A). Like the PSPs triggered in LG by Ints A and C, the PSP amplitude in compartment 3 increased when the LG model was hyperpolarized and decreased when it was depolarized. The sensitivity of the 
Figure 9. Model of the rectifying synapse between a mechanosensory neuron and LG. $A$, Diagram of the model. Compartments $1-6$ represent the LG neuron; an additional compartment represents the presynaptic region of the $M S I$. The diode symbol (for symbol descriptions, see Fig. 13) represents the rectifying electrical synapse between the MSI and LG; resistance symbols repLG neurite. Hatched compartments have voltage-sensitive currents (Hodgkin-Huxley inward and outward currents); open compartments are passive. Compartments where current was injected and/or responses were calculated are indicated by " $I$ " and " $V$ " electrodes, respectively. $B$, Current flow through the rectifying electrical synapse (middle) and responses of LG compartment 3 (bottom) to current steps $(-200,-100,0,100$, and $200 \mathrm{nA})$ injected into compartment 3 , and to an MSI spike (top). Impulse, synaptic current, and realigned EPSPs are shown at higher gains at right. Broken lines show postsynaptic responses when voltagesensitive conductances in compartments 4-6 were set to 0 and the injected currents $(-200,-100,0,50$, and 80 nA) were adjusted to give the same steady-state potential shifts in compartment 3. resent the longitudinal resistance in the
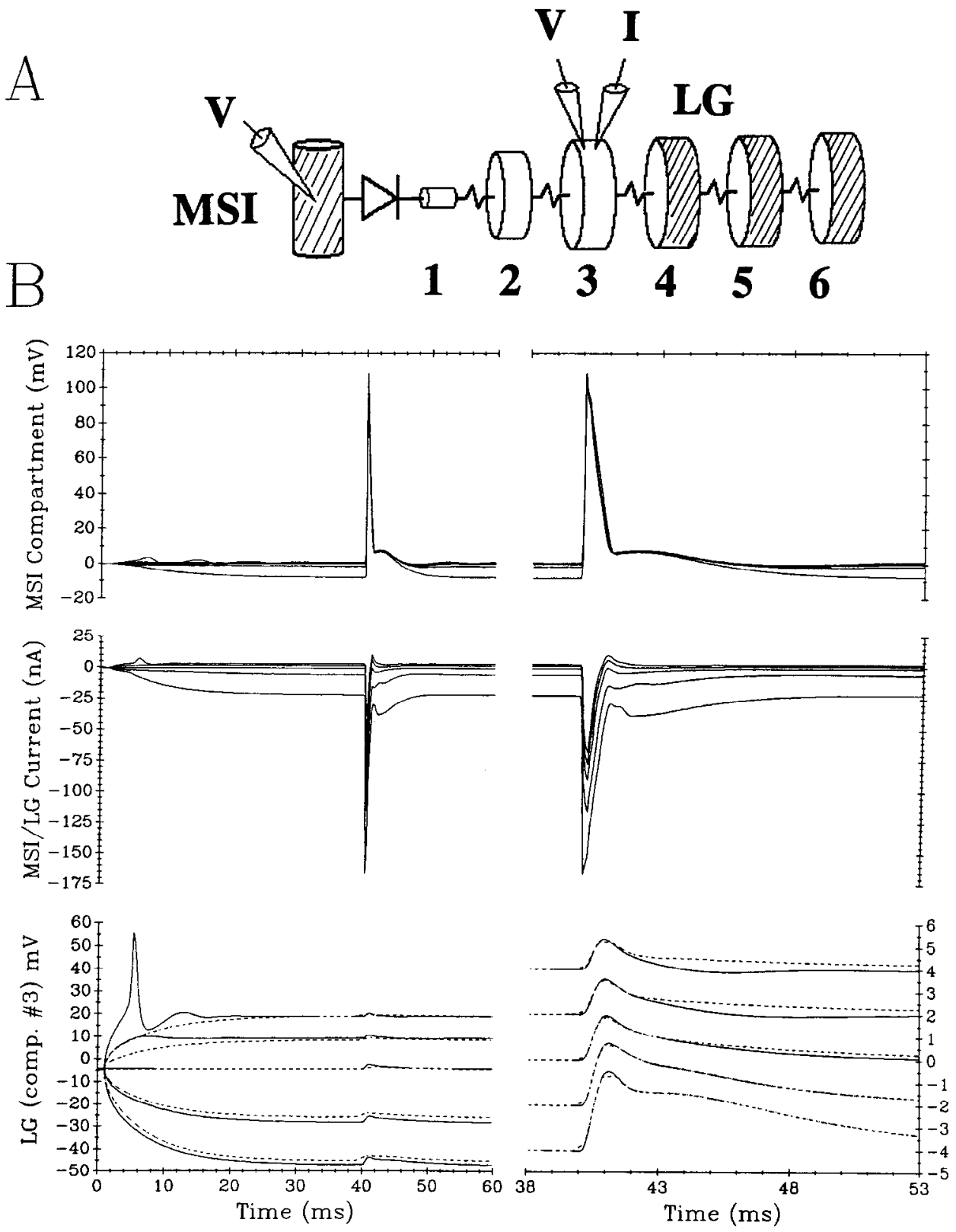

PSP to shifts in the compartment potential varied between $+1.7 \% / \mathrm{mV}$ for hyperpolarizations and $-1.4 \% / \mathrm{mV}$ for depolarizations. In addition, hyperpolarization of the LG model caused the late, slow phase of the PSP to increase in amplitude and duration, whereas depolarization reduced it.

The variation in PSP amplitude and wave form with PSP results from corresponding changes in the amplitude and duration of current through the rectifying synapse (Fig. $9 B$, middle). When the $\mathrm{LG}$ model was hyperpolarized to $-40 \mathrm{mV}$ below rest potential, the peak transsynaptic current evoked by a presynaptic impulse increased by $63 \%$ from its amplitude at rest, from $-88.5 \mathrm{nA}$ to $-143.5 \mathrm{nA}$. Part of this increase in peak synaptic current resulted from a $36 \%$ increase in the potential difference across the synapse at the peak of the presynaptic impulse.

The duration of the synaptic current pulse produced by a presynaptic impulse was longer when the LG compartments were hyperpolarized and shorter when they were depolarized. Changes in the duration of the high-conductance state of the rectifying synapse during the impulse account for much of these current changes. Postsynaptic shifts in resting potential change the level on the LG impulse where the synaptic rectification threshold is crossed; hyperpolarization causes more and depolarization causes less of the LG impulse to be above threshold. As a result, postsynaptic hyperpolarization "forward-biases" the synaptic rectifier into the high-conductance state for a longer period, and also increases the driving force across the synapse. Both factors increase the duration and amplitude of synaptic current. Conversely, postsynaptic depolarization "reverse-biases" the rectifier and reduces both the period of high synaptic conductance and the driving force for synaptic current during the impulse.

The depolarizing afterpotential that followed the presynaptic impulse significantly increased the duration of the PSP, es- 
pecially when the LG model was strongly hyperpolarized. The 50-mV postsynaptic hyperpolarization kept the transsynaptic potential above the rectification threshold during the depolarizing afterpotential and produced a large, slow synaptic current after the impulse. This current created a prolonged depolarization on the falling phase of the PSP. Smaller postsynaptic hyperpolarizations and depolarizations of the LG model brought the transsynaptic potential below rectification threshold during the depolarizing afterpotential and caused the late synaptic currents to be smaller and the repolarization following the PSP to be more rapid.

Activation of delayed rectifier conductances might also contribute to the reduction in PSP amplitude produced by postsynaptic depolarization. We addressed this question by setting the voltage-sensitive conductances in the LG model to 0 and calculating PSPs as before when the postsynaptic compartments were held at the previous hyperpolarized and depolarized levels. No change occurred at any of these levels in the peak PSP amplitudes (Fig. 9B, bottom, broken lines) from the values obtained when the voltage-sensitive conductances were operative (Fig. 9B, bottom, solid lines). Instead, depolarization activated delayed-rectifier conductances that reduced the late component of the LG response, and made the entire response more phasic. The voltage-sensitive conductances had little effect on the initial PSP amplitude because they are proximal to both the synaptic input site and the recording site, and because the transsynaptic current pulse has a short duration relative to the postsynaptic membrane time constant. The pulse was nearly over within 1 msec, whereas the membrane time constant of the LG model was $7.5 \mathrm{msec}$ when the voltage-sensitive conductances were set to 0 , and approximately half that figure when the conductances had their normal values (Table 1).

PSCs modulated by shifts in model LG potential. The amplitude of model PSCs also increased when the LG model was hyperpolarized and decreased when it was depolarized. PSCs were calculated when compartment 3 's potential was held under voltage clamp at hyperpolarized and depolarized levels (Fig. 10). Like the PSCs recorded in LG in response to Int $C$ spikes (Fig. 6D), the model LG PSCs each had a large, early, and brief transient phase and a smaller, later slow phase. Both phases were increased by postsynaptic hyperpolarization and decreased by depolarization.

Voltage sensitivity of summated PSPs. Repetitive activation of impulses in the MSI compartment evoked a series of EPSPs in the LG compartment (Fig. 11) that resembles the LG EPSPs

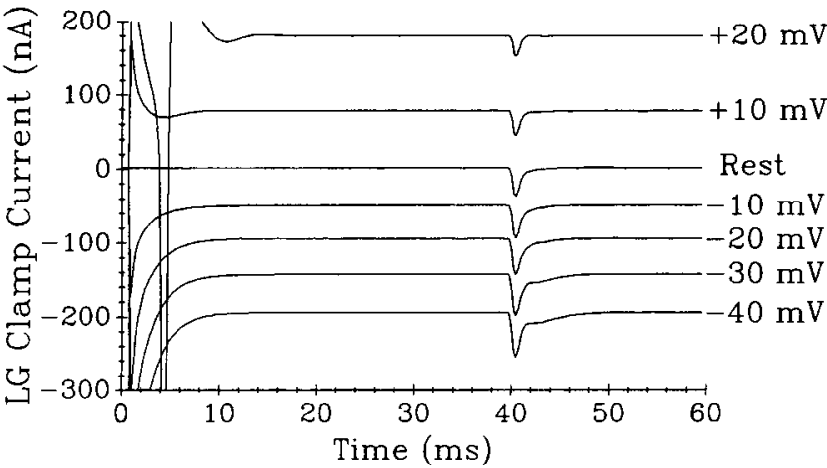

Figure 10. PSCs in compartment 3 evoked by an MSI spike when the compartment was held under voltage clamp at $+20,+10,0,-10,-20$, -30 , and $-40 \mathrm{mV}$ from rest potential.

evoked by the train of Int $\mathrm{C}$ impulses (Fig. 6C). Summation of the model EPSPs was enhanced by summation of the depolarizing afterpotentials in the train of presynaptic impulses. This created a sustained presynaptic depolarization of nearly $10 \mathrm{mV}$ (Fig. 11, top) that contributed to a sustained flow of current across the synapse (Fig. 11, middle). The synaptic current was also enhanced by the slow recovery kinetics of the rectifying synapse, which kept it in the high-conductance state nearly half of the short interval between impulses.

Polarization of the LG compartments affected the later, summated synaptic potential much more than the first EPSP. Model EPSPs were calculated each time after the LG model's potential had been shifted to one of the levels obtained before in Figure 9B. Postsynaptic hyperpolarization increased the driving force for the sustained synaptic current and the duration of the high transsynaptic conductance produced by each impulse. Transsynaptic current is proportional to the product of these factors, and so was increased dramatically. Postsynaptic depolarization had the opposite effect. These factors increased the sensitivity of the final PSP in the train to shifts in postsynaptic membrane potential. Whereas the sensitivity of the first PSP in the train was $-1.4 \% / \mathrm{mV}$ for depolarizations and $+1.7 \% / \mathrm{mV}$ for hyperpolarizations, the sensitivity of the final PSP was $-1.8 \% / \mathrm{mV}$ at the most depolarized level and $+4.3 \% / \mathrm{mV}$ at $-25 \mathrm{mV}$ from rest. The increased sensitivity of the late PSPs to membrane potential shifts is similar to that seen in the compound EPSP evoked by root shock (Fig. 7) and the summated PSPs produced by the high-frequency train of Int $\mathrm{C}$ impulses (Fig. 6).

Table 1. Parameter values for the electrical model of the rectifying electrical synapse between an MSI and LG

\begin{tabular}{|c|c|c|c|c|c|c|c|c|}
\hline Compartment & $R$ & $T$ & $E_{\mathrm{Na}}$ & $E_{\mathrm{K}}$ & $E_{\mathrm{L}}$ & $G_{\mathrm{NaMax}}$ & $G_{\mathrm{KMax}}$ & $R_{i-j}$ \\
\hline \multicolumn{9}{|l|}{ MSI } \\
\hline 7 & 0.5 & 2 & 100 & -7 & 2 & 2000 & 300 & \\
\hline \multicolumn{9}{|l|}{ LG } \\
\hline 1 & 20 & 7.5 & 115 & -8 & -4 & 0 & 0 & \\
\hline 2 & 4 & 7.5 & 115 & -8 & -4 & 0 & 0 & $R_{1-2}, 0.6$ \\
\hline 3 & 1.5 & 7.5 & 115 & -8 & -4 & 0 & 0 & $R_{2-3}, 0.15$ \\
\hline 4 & 0.8 & 7.5 & 115 & -8 & -4 & 500 & 150 & $R_{3-4}, 0.06$ \\
\hline 5 & 0.8 & 7.5 & 115 & -8 & -4 & 1000 & 300 & $R_{4-5}, 0.04$ \\
\hline 6 & 0.8 & 7.5 & 115 & -8 & -4 & 1000 & 300 & $R_{\mathrm{5}-6}, 0.04$ \\
\hline
\end{tabular}

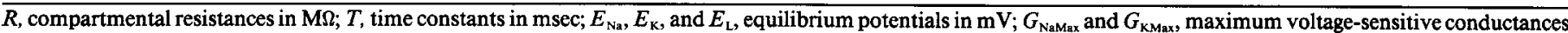
in $\mu \mathrm{S} ; R_{i-j}$, coupling resistances between LG compartments in $\mathrm{M} \Omega$. 

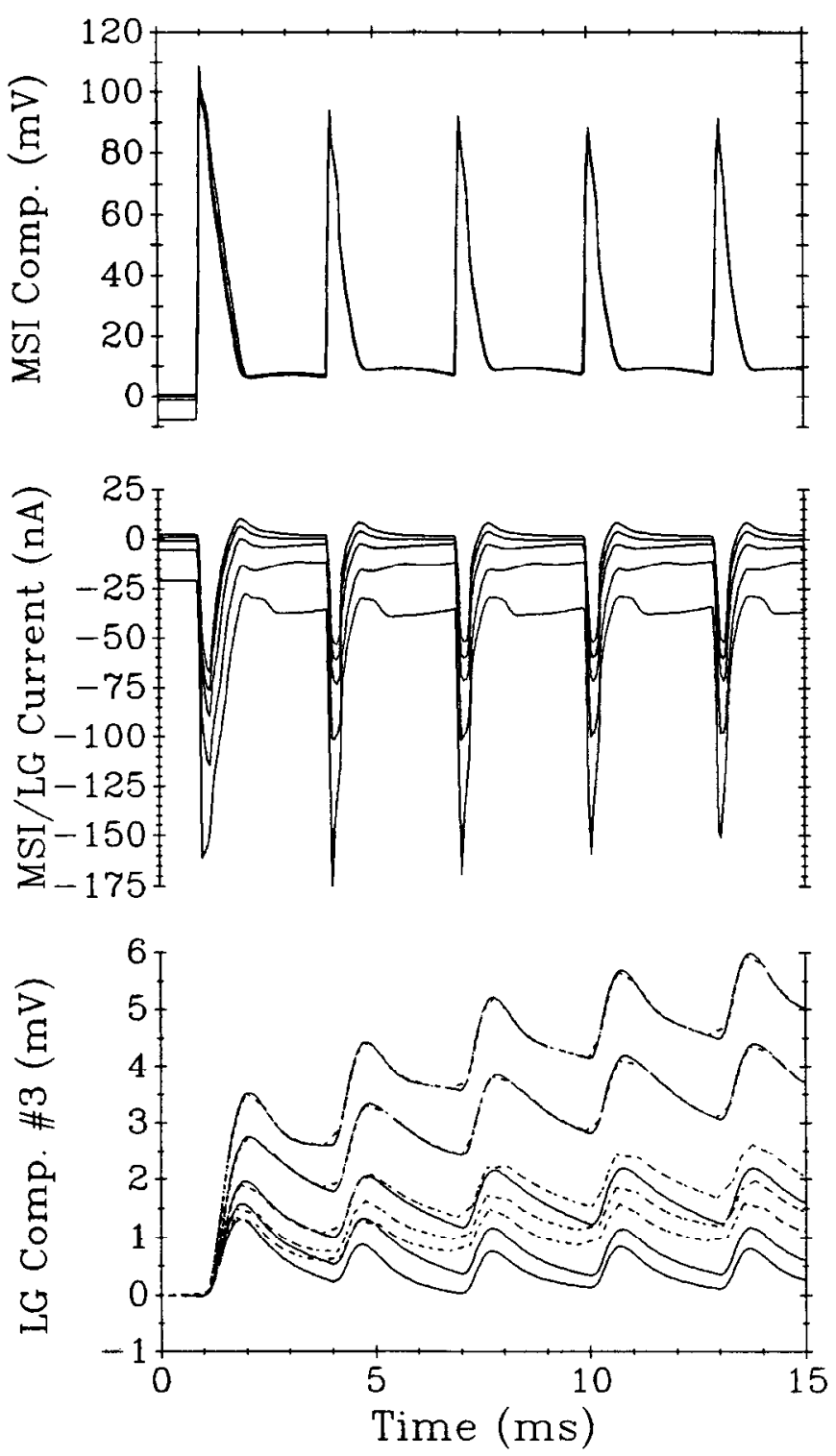

Figure 11. Summating PSPs in LG compartment 3 in response to impulses in MSI compartment and hyperpolarizing and depolarizing current steps in compartment 3. Impulses were evoked in MSI compartment $40 \mathrm{msec}$ after onset of current steps $(-200,-100,0,100$, and $200 \mathrm{nA})$ that evoke the same potential shifts as in Figure 9B. Top, Impulses in MSI compartment. Middle, Current through rectifying electrical synapse. Bottom, Summating EPSPs in compartment 3 with baselines aligned with $0 \mathrm{mV}$. EPSP amplitude varies with the negative shift of the compartmental potential. Solid lines, EPSPs calculated with maximum voltage-sensitive conductances in LG axonal compartments (46) set to values in Table 1; broken lines, PSPs calculated with voltagesensitive conductances set to 0 .

Both the forward- and reverse-bias effects on the synaptic rectifier and the delayed rectification associated with voltagesensitive conductances in the LG axonal compartments mediate the changes in summated PSP amplitudes caused by potential shifts. The separate contribution of the synaptic rectifier mechanism was assessed by removing the voltage-sensitive conductances from the axonal compartments and calculating the model's response to the same series of five presynaptic impulses (Fig. 11 , bottom, broken lines). Depolarizations of $12 \mathrm{mV}$ and 20 $\mathrm{mV}$ reduced the summated (fifth) PSP from the value at rest
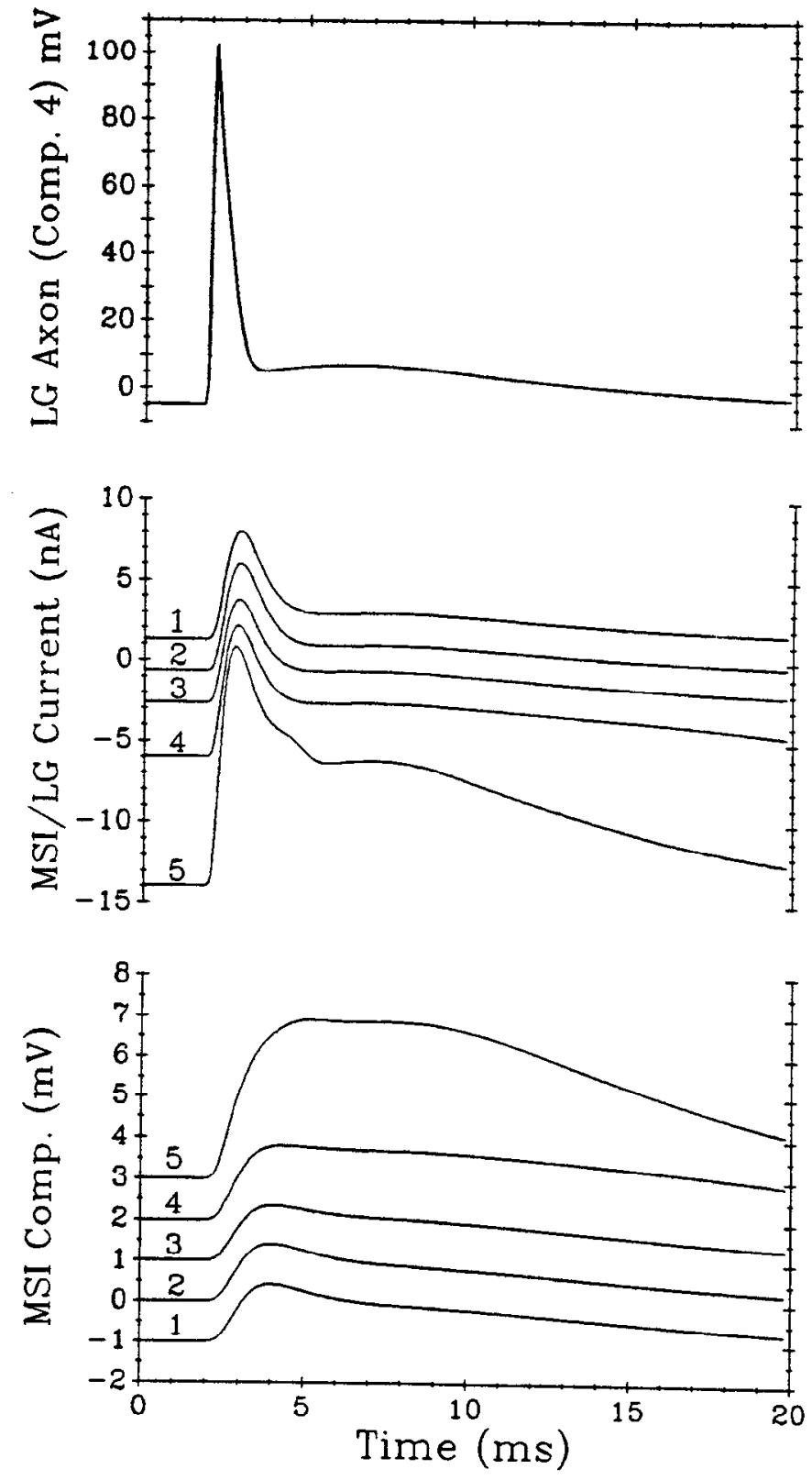

Figure 12. Modulation of antidromic synaptic potentials by potential shifts in the MSI compartment. Top, Antidromic impulse in compartment 4 of LG model. Middle, Synaptic currents produced by an antidromic impulse when MSI was at different MSI holding potentials maintained by injected currents. Holding potentials ( $\mathrm{mV}$ from rest potential) were as follows: trace $1,12 \mathrm{mV}$; trace $2,0 \mathrm{mV}$; trace $3,+11 \mathrm{mV}$; trace 4, +22 mV; trace 5, +32 mV. Bottom, Antidromic synaptic potentials amplified and aligned with baselines separated by $1 \mathrm{mV}$. Identically numbered traces at the middle and bottom are from the same simulation. The MSI compartment was assumed to be passive in these calculations.

potential by $26.5 \%$ and $46.5 \%$, respectively. Hyperpolarizations of 18 and $41 \mathrm{mV}$ produced the same summated PSP amplitudes as before, which were $69 \%$ and $131 \%$, respectively, above the value at rest. Addition of the voltage-sensitive conductances did not affect the hyperpolarized PSPs, but did significantly reduce the summated PSPs at rest and when the LG model was depolarized (Fig. 11, bottom, solid lines).

Modulation of antidromic synaptic potentials by potential shifts in the MSI compartment. Model antidromic synaptic potentials 
were affected by presynaptic potential shifts in the same manner as antidromic synaptic potentials in Int A (Fig. 4C) and Int C. Antidromic impulses were excited in the axonal compartments of the LG model, and the resulting antidromic synaptic potentials were calculated in the presynaptic MSI compartment, which was assumed to be passive (Fig. 12). As in both Int $A$ and Int $\mathrm{C}$, the model antidromic synaptic potentials had both an initial rapid phase and a later slow phase. Both phases increased significantly in amplitude when the MSI was depolarized and decreased slightly when it was hyperpolarized. The greatest change in the model antidromic synaptic potential occurred when the MSI compartment potential varied around the rectification threshold of $25 \mathrm{mV}$. Dcpolarization of the MSI compartment above the rectification threshold increased the transsynaptic conductance and enabled the LG depolarizing afterpotential to drive more current through the synapse and add to the antidromic synaptic potential. The falling phase of the model antidromic synaptic potential was then prolonged, much like the antidromic synaptic potentials recorded in Int $\mathrm{A}$ and Int $\mathrm{C}$ when those cells were similarly depolarized.

One difference between the model antidromic synaptic potential and those recorded in Ints $A$ and $C$ is that the model potential calculated when the MSI was strongly depolarized was shorter in duration and peaked earlier than the corresponding recorded potentials. This difference results in part from the shorter depolarizing afterpotential following the model LG spike, and the absence from the model of some of the low-pass signal filtering that occurs experimentally as the antidromic spike passes through the dendritic and axonal cable structures between the two recording sites.

Finally, it seemed possible that voltage-sensitive conductances might be activated when the MSI was depolarized, and that these might contribute to the enhanced antidromic synaptic potential. However, we found that addition of voltage-sensitive (Hodgkin-Huxley) sodium and potassium conductances to the MSI compartment reduced and shortened the antidromic synaptic potential when the compartment was tonically depolarized by as little as $6 \mathrm{mV}$ (not shown). This reduction occurred because tonic depolarization activated the potassium (delayed-rectifier) conductance, which shunted the antidromic synaptic potential. Additional depolarizing current caused the MSI to spike repetitively.

\section{Discussion}

Rectification at the afferent electrical synapses onto $L G$. The synaptic relationships of the three MSIs to LG are similar and are consistent with the presence of rectifying electrical synapses between each MSI and LG. In each case, depolarization of the presynaptic neuron strengthened the coupling between the two cells (measured either as PSP amplitude, as antidromic synaptic potential amplitude, or as the steady-state voltage drop across the synapse), and hyperpolarization of the cell weakened it. Conversely, depolarization of the postsynaptic cell (LG) weakened the coupling, and hyperpolarization strengthened it. These results are similar to those obtained with the model of the rectifying electrical synapse (Figs. 9-12) and to those obtained from similar experiments on the electrically rectifying giant motor synapse between LG and MoG (Furshpan and Potter, 1959a; Edwards, 1990a,b).

Like the PSPs evoked by MSI impulses, all components of the LG EPSP response to root shock increased when LG was hyperpolarized and decreased when it was depolarized. The
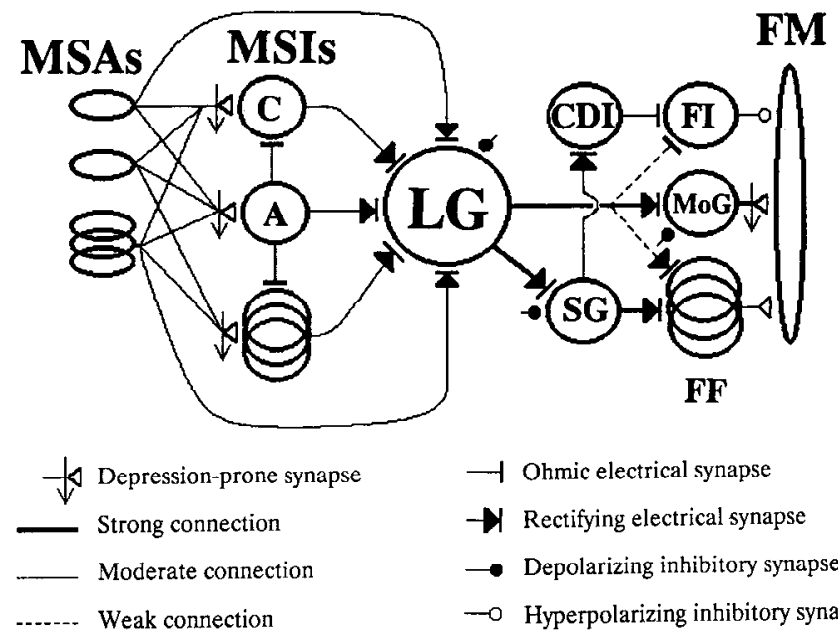

- Ohmic electrical synapse
$\rightarrow$ Rectifying electrical synapse
$\rightarrow$ Depolarizing inhibitory synapse
$\rightarrow$ Hyperpolarizing inhibitory synapse

Figure 13. A diagram of the LG escape circuit showing electrically rectifying and depolarizing inhibitory synapses. $M S A s$, mechanosensory afferents; $M S I s$, mechanosensory interneurons; $C$ and $A$, Ints $C$ and $\mathrm{A}$; $L G$, lateral giant interneuron; $M O G$, motor giant motor neuron; $C D I$, corollary discharge interneuron; $F I$, fast flexor inhibitor motor neuron; $S G$, segmental giant interneuron; $F F$, fast flexor motorneurons; $F M$, fast flexor muscle.

$\alpha$-component is produced by electrical synapses from mechanosensory afferents, whereas the later components are produced by di- and polysynaptic pathways involving the MSIs that also end at electrical synapses onto LG (Krasne, 1969; Zucker, 1972; Winc and Krasnc, 1982; Fricke, 1984). The evidence presented here suggests that all of these electrical synapses also rectify: coupling between the presynaptic mechanosensory afferent or MSI and LG increases when LG becomes more negative with respect to the presynaptic terminal and decreases when LG becomes more positive.

Origin of the $\gamma$-PSP component. The $\alpha$ - and $\beta$-components of the root-evoked PSP in LG are produced by the phasic discharge of mechanosensory afferents and MSIs, respectively (Krasne, 1969; Zucker, 1972). The $\gamma$-component is a much longer-lasting wave of depolarization that presumably results from the summation of PSPs evoked by the longer-lasting discharge of some of those same MSIs. Int C is clearly one of those, and the summating PSPs in LG produced by its high-frequency response (Fig. 6C) shows how it contributes to the $\gamma$-component. This conclusion is reinforced by the simulation of LG's response to the high-frequency discharge of the MSI (Fig. 11). The simulations show that spread of the depolarizing afterpotential across the synapse during high-frequency presynaptic discharges contributes significantly to the late, tonic depolarization of LG at the time of the $\gamma$-component. Presumably, other cells with firing patterns and depolarizing afterpotentials like Int C's will simultaneously make similar contributions to the $\gamma$-component.

The function of rectifying electrical synapses in the escape circuit. Rectifying synapses serve several ends in the escape circuit. First, they transmit rapidly. Speed is essential in the escape response, which launches the animal off the substrate within 15-20 msec of contact with the stimulus. Second, they are unidirectional, which prevents unwanted antidromic excitation of the escape circuit. This is necessary because many of the postsynaptic interneurons ( $\mathrm{LG}, \mathrm{SG}, \mathrm{MoG}$ ) are very large and have favorable size relationships for electrical coupling with their presynaptic drivers. For cxample, we found that, when Int A was sufficiently depolarized, the large antidromic synaptic 
potential evoked there by an LG impulse could trigger a spike in Int A (E. M. Leise, unpublished observations). Such a spike would tend to reexcite both LG and the higher-order MSIs, including Int $\mathrm{C}$. This does not normally happen because Int $\mathrm{A}$ is sufficiently depolarized to move the rectifying synaptic conductance into the high state only during its own spike. In a similar vein, rectification at the giant motor synapse allows either of the two different presynaptic escape command neurons, LG and MG, to excite MoG without having that cell produce unwanted antidromic excitation of the other command neuron. Third, rectifying synapses prevent the LG's response to active inputs from being antidromically shunted by low-resistance contacts that are currently quiescent (Krasne, personal communication). This allows LG to receive convergent synaptic contacts from numerous small afferents and large interneurons, and to be excited by any subset of those when the others are not active. Finally, transmission through rectifying electrical synapses can be modulated by shifts in the pustsynaptic cell's membrane potential.

Depolarizing inhibition at the rectifying electrical synapses. Depolarization of LG produced by the afferent synaptic input has both an excitatory and an inhibitory effect on the cell's response. Phasic mechanosensory stimuli that produce a rapidly depolarizing $\beta$-PSP can evoke a single LG impulse (Krasne, 1969). When an impulse fails to occur, however, the $\beta$-component is followed by the $\gamma$-PSP, a sustained depolarization lasting more than 30 mscc. Int A PSPs evoked during the $\beta$ and $\gamma$-components are attenuated between $50 \%$ and $80 \%$ (Krasne and Wine, 1987; Krasne et al., 1990; Vu and Krasne, unpublished observations). A large part of the attenuation is caused by a picrotoxin-sensitive, postexcitatory inhibition that is excited by the same strong mechanosensory stimuli that excite input to LG (Vu and Krasne, unpublished observations).

The results presented here show that depolarization (such as the $\gamma$-PSP) substantially reduces LG's response to mechanosensory afferent and MSI inputs by reducing excitatory synaptic current through the rectifying electrical synapses. Electrical rectification allows significant synaptic current to flow when the rectifier is forward biased, which normally occurs only during a presynaptic spike. Synaptic current evoked by a presynaptic spike is reduced when the postsynaptic depolarization reduces the transsynaptic driving force. This occurs when LG is depolarized during $\beta$ - and $\gamma$-PSPs by as much as $12 \mathrm{mV}$ at the initial segment, and by more at the tips of the dendrites (Krasne, 1969; Zucker, 1972). If the presynaptic impulses reach $100 \mathrm{mV}$ above LG's rest potential, the driving force for synaptic current produced by the impulses will be reduced by the amount of the postsynaptic potential, or about $12 \%$. The depolarizing afterpotential that follows each impulse does not exceed $12 \mathrm{mV}$ even during a high-frequency train of impulses; the driving force produced by the depolarizing afterpotentials should be greatly reduced or even eliminated by a large $\beta$ - or $\gamma$-PSP.

Postsynaptic depolarization also reduces synaptic current by increasing the level of presynaptic depolarization required for the rectifying synapse to enter the high-conductance state. As a result, the high-conductance state starts later and ends earlier during a presynaptic impulse, and so shortens the effective period of the driving force. Postsynaptic depolarization may prevent the transsynaptic potential from ever reaching rectification threshold during the depolarizing afterpotential, so that the synapse stays in the low-conductance state.

These two effects of postsynaptic depolarization, a reduction in the transsynaptic driving force and a shortened or eliminated period of high transsynaptic conductance, combine to reduce further input through rectifying electrical synapses. They also select for phasic inputs, because the slower synaptic currents created by depolarizing afterpotentials are more severely attenuated than the phasic synaptic currents produced by impulses. Activation of the delayed-rectifier conductance also preferentially reduces low-frequency responses to synaptic input. These effects combine to reduce the very phasic $\alpha$-PSP component by $1.0 \% / \mathrm{mV}$ of postsynaptic depolarization imposed and measured at the initial segment, which should be not much greater than the potential in the distal dendrites. The less phasic $\beta$-component is decreased by $2.4 \% / \mathrm{mV}$, and the slower $\gamma$-PSP, by $2.75 \% /$ $\mathrm{mV}$.

Functionally, the relevant question is how much will a PSP that falls on top of an earlier PSP be reduced as a result of that earlier postsynaptic depolarization. This will depend both on the component whose reduction is being evaluated and on the component of the previously produced PSP on which it falls. For example, a large $\alpha$-component that measures $3 \mathrm{mV}$ at the initial segment will be $25 \mathrm{mV}$ or more in the dendrites (Zucker, 1972; E. Vu, personal communication; Edwards, unpublished observations), and so would be expected to reduce the $\alpha-, \beta-$, and $\gamma$-components of a second PSP by as much as $25 \%, 60 \%$, and $68 \%$, respectively. The corresponding reductions caused by the $\beta$-component of the first PSP should be larger still because the $\beta$-component is so much larger than the $\alpha$-component. The $\gamma$-component is smaller in the dendrites than the $\alpha$-component [being slow, its distal-to-proximal attenuation is small and so it becomes larger than the $\alpha$-component in the initial segment (Zucker, 1972; Vu, personal communication; Edwards, unpublished observations)] and so can be expected to cause less reduction in a superimposed PSP than the $\alpha$-component.

These last conclusions make it apparent that several mechanisms enable LG to be highly sensitive to phasic inputs and insensitive to gradually increasing or tonic inputs (Wine and Krasne, 1982). First, depolarization of LG produced by earlier inputs or by the depolarizing afterpotential following an LG impulse will reverse-bias the rectifying electrical input synapses and reduce their synaptic currents by the mechanisms described above. This has a smaller effect on the phasic currents produced by the presynaptic impulses and a greater effect on the slower currents produced by the presynaptic depolarizing afterpotentials. Second, depolarization will activate the delayed-rectifier conductances and decrease the input resistance of LG. This will also preferentially discriminate against more tonic inputs. Third, postexcitatory inhibition is activated on the falling phase of the $\beta$-component and strongly reduces inputs that occur at that time (Krasne and Wine, 1987; Krasne et al., 1990; Vu and Krasne, unpublished observations). Postexcitatory inhibition appears to be mediated by a depolarizing chemical inhibition located on the distal LG dendrites, where it shunts nearby incoming synaptic current. Fourth, membrane sodium conductance in LG may inactivate in response to maintained depolarization, which would increase LG's spike threshold. In MoG, tonic depolarizations produce significant sodium inactivation, and this serves as a mechanism of depolarizing inhibition of the cell's response (Edwards, 1990a). Finally, the low input resistance and short time constant of LG cause synaptic charge to redistribute rapidly through the large cell's structure and then to leak out (Zucker, 1972; Wine and Krasne, 1982).

Ubiquity of electrical rectification and depolarizing inhibition 
in the $L G$ escape circuit. The results presented here and recently elsewhere (Fraser and Heitler, 1989, 1991; Heitler and Fraser, 1989) demonstrate that most of the electrical synapses in the sensory afferent to motoneuron path of the escape circuit are rectifying synapses (Fig. 13). In addition to the synapses onto the LG from mechanosensory afferents and from the interneurons, they include synapses from LG to SG in each hemi-ganglion, from SG to the fast flexor motoneurons and to a corollary discharge interneuron (Fraser and Heitler, 1989), from LG to the fast flexor motoneurons (K. Fraser and W. J. Heitler, unpublished observations), and from LG to MoG (Furshpan and Potter, 1959a). The major exceptions appear to be the septate junctions between adjacent LGs and the electrical synapses between contralateral LGs in the same segment, all of which are ohmic electrical synapses, and act to unite the LGs in each abdominal hemi-segment into a single functional unit (Watanabe and Grundfest, 1961).

Another general feature of the escape circuit is the ubiquity of depolarizing inhibition, especially on cells postsynaptic to rectifying electrical synapses (Fig. 13). In addition to the depolarizing inhibition of LG described here and elsewhere (Roberts, 1968; Krasne et al., 1990; Vu and Krasne, unpublished observations), MoG (Hagiwara, 1958, Furshpan and Potter, 1959b) and SG (Fraser and Heitler, 1989) also receive d-IPSPs. Finally, the ability of postsynaptic potential changes to modulate transmission through rectifying electrical synapses has also been seen in the leech (Friesen, 1985), and it may occur at similar synapses in vertebrates, including those in the hatchetfish (Auerbach and Bennett, 1969) and lamprey (Ringham, 1975).

\section{References}

Auerbach AA, Bennett MVL (1969) A rectifying electrotonic synapse in the central nervous system of a vertebrate. J Gen Physiol 53:211237.

Calabrese RL, Kennedy D (1974) Multiple sites of spike initiation in a single dendritic system. Brain Res 82:316-321.

Edwards DH (1990a) Mechanisms of depolarizing inhibition at the crayfish giant motor synapse. I. Electrophysiology. J Neurophysiol 64:532-540.

Edwards DH (1990b) Mechanisms of depolarizing inhibition at the crayfish giant motor synapse. II. Quantitative reconstruction. J Neurophysiol 64:541-550.

Edwards DH, Mulloney B (1984) Compartmental models of electrotonic structure and synaptic integration in an identified neurone. $\mathbf{J}$ Physiol (Lond) 348:89-113.

Edwards DH, Mulloney B (1987) Synaptic integration in excitatory and inhibitory crayfish motoneurons. J Neurophysiol 57:1425-1445.

Fraser K, Heitler WJ (1989) Thoracic output of crayfish giant fibres. II. The segmental giant neurone. J Comp Physiol A 166:125-132.

Fraser K, Heitler WJ (1991) Photoinactivation of the crayfish segmental giant neuron reveals a direct giant-fiber to fast-flexor connection with a chemical component. J Neurosci 11:59-71.

Fricke RA (1984) Development of habituation in the crayfish due to selective weakening of electrical synapses. Brain Res 322:139-143.
Friesen WO (1985) Neuronal control of leech swimming movements: interactions between cell 60 and previously described oscillator ncurons. J Comp Physiol A 156:231-242.

Furshpan EJ, Potter DD (1959a) Transmission at the giant motor synapses of the crayfish. J Physiol (Lond) 145:289-325.

Furshpan EJ, Potter DD (1959b) Slow post-synaptic potentials recorded from the giant motor fibre of crayfish. J Physiol (Lond) 145: 326-335.

Giaume C, Kado RT, Korn H (1987) Voltage-clamp analysis of a crayfish rectifying synapse. J Physiol (Lond) 386:91-1 12 .

Hagiwara S (1958) Synaptic potential in motor giant axon of the crayfish. J Gen Physiol 41:1119-1 128.

Heitler WJ, Fraser K (1989) Thoracic output of crayfish giant fibres. I. Pereiopod promotor motor neurones. J Comp Physiol A 166:117124.

Hodgkin AL, Huxley AF (1952) A quantitative description of membrane current and its application to conduction and excitation in nerve. J Physiol (Lond) 117:500-544.

Jaslove SW, Brink PR (1986) The mechanism of rectification at the electrotonic motor giant synapse of the crayfish. Nature 323:63-65.

Kennedy D (1971) Crayfish interneurons. Physiologist 14:5-30.

Krasne FB (1969) Excitation and habituation of the crayfish escape reflex: the depolarizing response in lateral giant fibers of the isolated abdomen. J Exp Biol 50:29-46.

Krasne FB, Wine JJ (1987) Evasion responses of the crayfish. In: Aims and methods in neuroethology (Guthrie DM, ed), pp 10-45. Manchester: Manchester UP.

Krasne FB, Vu ET, Lee SC (1990) The excitability of the crayfish lateral giant escape reaction: inhibitory control of the lateral giant dendrites. In: Frontiers in crustacean neurobiology (Wiese K, Krenz W-D, Tautz J, Reichert H, Mulloney B, eds), pp 316-322. Berlin: Birkhauser.

Obermayer M, Strausfeld NJ (1980) Silver staining cobalt sulfide deposits within neurons of intact ganglia. In: Neuroanatomical techniques. Insect nervous system (Strausfeld NJ, Miller TA, eds), pp 406-430. New York: Springer.

Ringham GL (1975) Localization and electrical characteristics of a giant synapse in the spinal cord of the lamprey. J Physiol (Lond) 251: 395-407.

Roberts AM (1968) Recurrent inhibition in the giant-fibre system of the crayfish and its effect on the excitability of the escape response. J Exp Biol 48:545-567.

Sigvardt KA, Hagiwara G, Wine JJ (1982) Mechanosensory integration in the crayfish abdominal nervous system: structural and physiological differences between interneurons with single and multiple spike initiating sites. J Comp Physiol A 148:143-157.

Stewart WW (1978) Functional connections between cells as revealed by dye-coupling with a highly fluorescent napthalimide tracer. Cell 14:741-749.

Van Harreveld A (1936) A physiological solution for freshwater crustaceans. Proc Soc Exp Biol Med 34:428-432.

Watanabe A, Grundfest $H$ (1961) Impulse propagation at the septal and commissural junctions of crayfish lateral giant axons. J Gen Physiol 45:267-308.

Wine JJ (1984) The structural basis of an innate behavioural pattern. J Exp Biol 112:283-319.

Wine JJ, Krasne FB (1982) The cellular organization of crayfish escape behavior. In: The biology of crustacea, Vol 4 (Sandeman DC, Atwood HL, eds), pp 241-292. New York: Academic.

Zucker RS (1972) Crayfish escape behavior and central synapses. I. Neural circuit exciting lateral giant fiber. J Neurophysiol 35:599-620. 